\section{Haemoptysis with a normal chest radiograph: how concerned should we be?}

\author{
Gene L Colice
}

In this issue of Thorax (see page 854), Thirumaran and colleagues describe their experience with a consecutive group of patients evaluated for haemoptysis who had a normal chest radiograph. ${ }^{1}$ Over a 4year period these investigators identified 270 patients who were evaluated for this problem. They made two important observations. First, they found that 26 of the 270 patients $(9.6 \%)$ had respiratory tract malignancies as the cause of the haemoptysis. Of these 26 respiratory tract malignancies, 20 were non-small cell lung cancer (NSCLC) and 2 were small cell lung cancer. Secondly, they performed both fibreoptic bronchoscopy (FOB) and chest CT on the majority of the 270 patients evaluated. Overall, at least $269 \mathrm{FOBs}$ and 257 CTs were performed to diagnose these 22 lung cancers. These observations lead to a series of fundamentally important questions for doctors to consider.

Why should haemoptysis with a normal chest radiograph be a concern? Haemoptysis can be massive and lifethreatening, by causing either airway compromise or haemodynamic insufficiency. Fortunately, haemoptysis is rarely massive. I work in a busy Intensive Care Unit (ICU; 19 beds and $>1500$ admissions per year) in a large urban hospital (907 beds and about 47000 admissions per year) and we see probably only 1-2 cases of massive, life-threatening haemoptysis per year. In the study by Thirumaran et al, of the 275 episodes of haemoptysis, none was massive; the majority were described as simply streaks of bleeding. Although doctors should be aware of the risk of massive haemoptysis, most cases of haemoptysis represent minor bleeding which stops spontaneously. The real concern about haemoptysis with a normal chest radiograph is understanding the cause. As Thirumaran and colleagues point out, haemoptysis should be considered a possible signal of an underlying serious illness, particularly lung cancer. Doctors should

Correspondence to: Dr Gene L Colice, Washington Hospital Center, 110 Irving St, NW, Washington, DC 20010, USA; Gene.Colice@Medstar.net not be lulled into believing that lung cancer has been firmly excluded by the finding of a normal chest radiograph. Quekel and colleagues found that in 49 of 259 patients (19\%) with NSCLC presenting as a nodular lesion on chest radiograph the cancer had been "missed"- - that is present but not identified by the radiologist-on previous chest radiographs. ${ }^{2}$ Shah et al also documented cases of NSCLC "missed" during an initial chest radiograph. " "Missed" cases of NSCLC on chest radiograph were usually small tumours in the upper lobes with often superimposed structures, although endobronchial cancers can also be "missed" on a chest radiograph. The 22 lung cancers documented in this study by Thirumaran et al were "missed" by chest radiography. The consequence of an NSCLC "missed" on chest radiograph is a delay in diagnosis with possibly failure to provide a surgical cure. $^{2}$

How should general practitioners (GPs) consider haemoptysis as a presenting symptom? Haemoptysis, even with a normal chest radiograph, should be considered a signal of lung cancer. This is an important message because it has been estimated that a GP in the UK might see a new lung cancer case only once in every 8 months. ${ }^{4}$ Respiratory symptoms are common presenting complaints to GPs, but respiratory symptoms are usually due to benign causes. Consequently, GPs probably do not consider lung cancer often in their daily practice. However, of all the respiratory symptoms seen in general practice, haemoptysis seems to be most clearly linked to lung cancer. ${ }^{4}$ One weakness in the study by Thirumaran et al is that it is unclear how many patients in their catchment area actually presented to their GP with haemoptysis. I suspect that the number of patients presenting with haemoptysis to GPs (with either an unremarkable chest radiograph or with no chest radiograph obtained) in the West Yorkshire region during the years of this study was probably (substantially) higher than the number actually referred for evaluation at the Dewsbury and District Hospital. Education about the concerns related to haemoptysis at the local level is clearly needed.

How accurately does haemoptysis with a normal chest radiograph predict an occult lung cancer? In this series, there were 22 cases of lung cancer in 270 patients, reflecting a prevalence of lung cancer of $8.2 \%$ for this clinical scenario. ${ }^{1}$ Others have reported that the prevalence of lung cancer for this clinical scenario might be $5-6 \%{ }^{5}$ It is interesting to consider the reported prevalence of lung cancer in patients presenting with haemoptysis and a normal chest radiograph in the context of the ongoing controversy over screening for lung cancer. In studies published since 2004 using low dose CT (LDCT) for lung cancer screening, performed in asymptomatic populations older than age 40 usually with some smoking history, the baseline prevalence of lung cancer varied from $1.3 \%$ to $2.8 \% .^{6-13}$ Clinicians accept the value of searching for a lung cancer in a patient presenting with haemoptysis and a normal chest radiograph, but not in an asymptomatic patient older than 40 with a smoking history. ${ }^{14}$ This observation suggests that perhaps the benefit of screening for lung cancer could be demonstrated in a subset of asymptomatic patients with a somewhat higher prevalence of lung cancer. The implications of this insight are important from a public health perspective. In the USA, the number of lung cancers continues to increase, most lung cancers present in an advanced stage, and the projected 5-year survival for lung cancer remains disappointingly low. ${ }^{14}$ Identifying lung cancer early enough with a safe screening test to allow curative surgical resection would seem to be an attractive strategy for addressing the lung cancer problem. The approach over the last decade to earlier identification of lung cancer has relied on using more sophisticated chest imaging techniques, for example LDCT versus chest radiography. Improved imaging alone, though, might not be the solution. Ongoing studies using LDCT to screen for lung cancer have also attempted to identify higher risk patients using smoking history. ${ }^{15}$ Other approaches to risk assessment besides age and smoking history are also being explored, such as analyses of exhaled breath volatiles. ${ }^{16}$ Improved imaging applied to patients with a known higher prevalence of disease is a promising approach to solving the lung cancer screening dilemma.

How should a doctor evaluate a patient presenting with haemoptysis and a normal chest radiograph? Thirumaran and colleagues performed both $\mathrm{FOB}$ and chest $\mathrm{CT}$ in $>90 \%$ of the patients who presented with 
haemoptysis and a normal chest radiograph. This approach seems clinically reasonable, because chest radiography "misses" small lung cancers and endobronchial lung cancers. However, the combined use of FOB and CT resulted in hundreds of procedures being performed, suggesting that a more efficient process for identifying lung cancers is needed. There are a variety of ways, such as cost-effectiveness analyses, to look at the efficiency of diagnostic strategies. One intriguing approach to compare the efficiency of diagnostic strategies is to calculate the number of tests needed to establish a diagnosis (NTND). This approach appeals to clinicians because it reflects how they solve problems on a daily basis. In a hypothetical analysis of a cohort of patients presenting with haemoptysis and a normal chest radiograph the NTND was calculated for various diagnostic approaches. ${ }^{5}$ Strategies employing either FOB or CT first, followed by the other test if non-diagnostic results were obtained, resulted in a substantial NTND, just as Thirumaran and colleagues reported. ${ }^{1}$ However, a novel approach incorporating the use of sputum cytology as an initial diagnostic test resulted in a substantially lower NTND than either the FOB or CT first approach, while still maintaining a high degree of diagnostic accuracy. Sputum cytology can identify endobronchial lung cancer with a reasonably low false-positive rate. An abnormal sputum cytology would be followed by FOB. An unremarkable sputum cytology could be followed by CT, or by serial chest radiographs.

Haemoptysis with a normal chest radiograph should raise concerns at several levels.
For the individual patient and their doctor, it should raise the suspicion of lung cancer. GPs see many patients with respiratory symptoms, most of which are due to relatively benign causes. Haemoptysis, though, seems to be a more accurate predictor of underlying lung cancer than other respiratory symptoms, such as cough. For the overall healthcare system, a clearer understanding of which patients would benefit from screening for lung cancer is obviously needed. It is curious that the baseline prevalence of lung cancer in LDCT screening trials ranges from $1.3 \%$ to $2.8 \%$, yet the value of lung cancer screening in the asymptomatic patient with a smoking history has not been established. Hopefully, the results of the National Lung Screening Trial, a 50000 patient study evaluating lung cancer mortality in patients undergoing screening with LDCT currently underway in the USA, will clarify this situation. Alternatively, perhaps concentrating on integrating other methods into the screening process, which would identify patients with a higher prevalence of lung cancer, might increase the value of LDCT screening. At present, clinical practice accepts that there are subsets of patients, such as those with haemoptysis and a normal chest radiograph, with an expected higher prevalence of lung cancer who warrant careful evaluation. Clinicians should be aware, though, that there are strategies for searching for lung cancer in these patients which seem to be more efficient than performing $\mathrm{CT}$ and $\mathrm{FOB}$ on every patient.

Competing interests: None.

Provenance and peer review: Commissioned; not externally peer reviewed.

Thorax 2009;64:829-830. doi:10.1136/thx.2009.113191

\section{REFERENCES}

1. Thirumaran $\mathbf{M}$, Sundar R, Sutcliffe $\mathbf{I M}$, et al. Is investigation of patients with haemoptysis and normal chest radiograph justified? Thorax 2009:64:854-6.

2. Quekel LGBA, Kessels AGH, Goei R, et al. Miss rate of lung cancer on the chest radiograph in clinical practice. Chest 1999;115:720-4.

3. Shah PK, Austin JHM, White CS, et al. Missed nonsmall cell lung cancer. Radiology 2003;226:235-41.

4. Hamilton W, Peters TJ, Round A, et al. What are the clinical features of lung cancer before the diagnosis is made? Thorax 2005;60:1059-65.

5. Colice GL. Detecting lung cancer as a cause of hemoptysis in patients with a normal chest radiograph. Chest 1997;111:877-84.

6. Diederich S, Thomas M, Semik M, et al. Screening for early lung cancer with low-dose spiral computed tomography. Eur Radiol 2004;14:691-702.

7. Swensen SJ, Jett JR, Hartman TE, et al. CT screening for lung cancer. Radiology 2005;235:259-65.

8. Gohagan JK, Marcus PM, Fagerstrom RM, et al. Final results of the Lung Screening Study, a randomized feasibility study of spiral CT versus chest $\mathrm{X}$-ray screening for lung cancer. Lung Cancer 2005; 47:9-15

9. Bastarrika G, Garcia-Velloso MJ, Lozano MD, et al. Early lung cancer detection using spiral computed tomography and positron emission tomography. Am J Respir Crit Care Med 2005;171:1378-83.

10. I-ELCAP. Survival of patients with stage I lung cancer detected on CT screening. N Engl J Med 2006;355:1763-71.

11. Blanchon T, Brechot JM, Grenier PA, et al. Baseline results of the Depiscan study. Lung Cancer 2007; 58:50-8

12. Infante $\mathbf{M}$, Lutman FR, Cavuto $\mathrm{S}$, et al. Lung cancer screening with spiral CT. Lung Cancer 2008;59:355-63.

13. Wilson DO, Weissfeld JL, Fuhrman CR, et al. The Pittsburg lung screening study (PluSS). Am J Respir Crit Care Med 2008;178:956-61.

14. Bach PB, Silvestri GA, Hanger M, et al. Screening for lung cancer. Chest 2007;132:S69-77.

15. van lersel CA, de Koning HJ, Draisma G, et al. Riskbased selection from the general population in a screening trial. Int J Cancer 2006;120:868-74.

16. Mazzone P. Progress in the development of a diagnostic test for lung cancer through the analysis of breath volatiles. J Breath Res 2008;2:1-6.

\section{Blood glucose: of emerging importance in COPD exacerbations}

\section{Emma H Baker, ${ }^{1}$ Derek Bell ${ }^{2}$}

Elevated blood glucose is a well recognised and common pathophysiological response

\footnotetext{
${ }^{1}$ St George's, University of London, London, UK;

${ }^{2}$ Imperial College, Chelsea and Westminster Hospital, London, UK
}

Correspondence to: Dr Emma Baker, Centre for Clinical Pharmacology, Division of Basic Medical Sciences, St George's, University of London, Cranmer Terrace, London SW17 ORE, UK; ebaker@sgul.ac.uk to acute illness. The underlying mechanisms include acute increases in hepatic glucose production and peripheral insulin resistance, driven by increases in glucocorticoids, catecholamines and proinflammatory cytokines. ${ }^{1}$ Acute hyperglycaemia can occur in any acutely unwell patient, irrespective of baseline glucose tolerance, if the illness is sufficiently severe. Acute hyperglycaemia is associated with poor outcomes from a wide range of acute illnesses including myocardial infarction, ${ }^{2}$ stroke, ${ }^{3}$ trauma ${ }^{4}$ and pneumonia. ${ }^{5}$

The data in relation to hyperglycaemia and acute exacerbations of chronic obstructive pulmonary disease (COPD) are now beginning to emerge. In a previous study, more than $50 \%$ patients with acute exacerbations of COPD had random blood glucose $\geqslant 7 \mathrm{mM}$ during their hospital stay. ${ }^{6}$ This retrospective study could not fully elucidate the relative contributions of acute illness, steroid therapy and underlying diabetes to development of hyperglycaemia. Nevertheless blood glucose $\geqslant 7 \mathrm{mM}$ was significantly associated with increased risk of death or prolonged hospital stay, and the absolute risk of this composite adverse outcome increased by $15 \%$ for each $1 \mathrm{mM}$ increase in blood glucose. 
It is also known that acute hypercapnic respiratory failure is an independent predictor of poor outcome for patients with COPD exacerbations. Non-invasive ventilation (NIV) reduces the likelihood of endotracheal intubation, treatment failure and mortality. ${ }^{7}$ Moretti and colleagues found that "metabolic complications" of COPD exacerbations, including hyperglycaemia defined as blood glucose $>11 \mathrm{mM}$, were independently associated with "late failure" ( $>48 \mathrm{~h})$ of NIV after initial success. ${ }^{8}$ In this study only $\sim 7 \%$ patients had blood glucose $>11 \mathrm{mM}$ and the relationship between more moderate hyperglycaemia $(>6-11 \mathrm{mM})$ and outcomes was not determined. Interestingly all of the patients with late NIV failure who had admission blood glucose $>11 \mathrm{mM}$ subsequently developed pulmonary infection, which may have contributed to NIV failure or death.

The study by Chakrabarti and colleagues in this issue of Thorax (see page 857) investigated the relationship between hyperglycaemia and NIV outcome prospectively in unselected COPD patients with acute hypercapnic respiratory failure. ${ }^{9}$ They found that hyperglycaemia, defined as random blood glucose $\geqslant 7 \mathrm{mM}$ within $24 \mathrm{~h}$ of hospital admission, was present in $50 \%$ patients, consistent with previous findings. ${ }^{6}$ NIV failure was significantly more common in patients with (34\%) than without (2\%) hyperglycaemia, and blood glucose was higher in those in whom NIV failed (mean (SD) 9.0 (3.2) $\mathrm{mM}$ ) than those in whom NIV was successful (7.0 (2.2) mM). This reinforces the concept that even moderate hyperglycaemia may be associated with poorer prognosis in acute illness.

It is not known whether hyperglycaemia is a direct cause of poor outcomes from COPD exacerbations or acts as a marker for other adverse prognostic factors such as treatment variation, comorbidity or severity of acute illness. In their prospective study, Chakrabarti and colleagues provide some new insights into the underlying mechanisms. They found that the association between hyperglycaemia and NIV failure in COPD exacerbations was not explained either by oral corticosteroid use immediately before admission or by underlying diabetes mellitus. Although animal studies have shown that respiratory acidosis causes glucose intolerance by inducing hepatic and peripheral insulin resistance, ${ }^{10}$ in this study hyperglycaemia predicted NIV outcomes independently of $\mathrm{pH}$ and was not merely a marker for low $\mathrm{pH}$. The effect of hyperglycaemia was also independent of
APACHE II (Acute Physiology and Chronic Health Evaluation II) score, a marker of illness severity.

Limitations of the study by Chakrabarti et al include small sample size and lack of information relevant to underlying glucose intolerance such as body mass index and previous episodes of hyperglycaemia during exacerbations. As they point out, glucose was only recorded once on admission and no attempt was made to quantify hyperglycaemia after admission or relate this to outcomes. The authors also do not describe the causes of NIV failure or death in their patients and so have missed an opportunity to explore a causative role for hyperglycaemia in NIV failure. Potential detrimental effects of hyperglycaemia include augmentation of inflammation and infection, and insulin resistance could increase muscle catabolism. Experimental hyperglycaemia induced an acute rise in interleukin-6 (IL-6), tumour necrosis factor $\alpha$ (TNF $\alpha$ ) and IL-18 that was inhibited by glutathione, indicating induction of proinflammatory cytokines via an oxidative mechanism. ${ }^{11}$ C-reactive protein was elevated in people with impaired glucose tolerance compared with those with normal glucose tolerance. ${ }^{12} 13$ In two separate studies of COPD exacerbations, acute hyperglycaemia and underlying diabetes were associated with increased isolation of multiple pathogens and Staphylococcus aureus ${ }^{6}$ and Gram-negative bacteria $^{14}$ from sputum. Hyperglycaemia is associated with elevated glucose concentrations in tissues and bronchial aspirates $^{15}$ where it may stimulate infection by enhancing bacterial growth ${ }^{16}$ and by promoting bacterial interaction with the airway epithelium. ${ }^{17}$ Hyperglycaemia also impairs both innate and adaptive immunity, suppressing the host response to infection. Skeletal muscle abnormalities are common in patients with COPD, and exacerbations requiring hospitalisation are associated with increasing muscle weakness ${ }^{18}$ and reduction in lean body mass. ${ }^{19}$ As insulin is an anabolic hormone, insulin resistance could exacerbate muscle loss during a COPD exacerbation. In support of this, patients with critical illness who did not receive insulin therapy had lower skeletal muscle total protein concentrations than those receiving pharmacological doses of insulin. ${ }^{20}$

The association between hyperglycaemia and poor outcomes raises the important question as to whether correction of hyperglycaemia with insulin could improve outcomes from COPD exacerbations. Initial enthusiasm for tight glycae- mic control in critical illness was generated by a single-centre, randomised controlled trial (RCT) of intensive insulin therapy compared with usual treatment in cardiothoracic patients requiring a stay in an Intensive Care Unit (ICU). ${ }^{21}$ Tight glycaemic control (blood glucose 4.4 $6.1 \mathrm{mM}$ ) reduced mortality by $42 \%$ compared with conventional glycaemic control (blood glucose 10-11.1 mM). In a second RCT by the same investigators in medical ICU patients, tight glycaemic control did not reduce mortality in the whole group but did reduce in-hospital mortality in those requiring a stay in the ICU for $\geqslant 3$ days. ${ }^{22}$ However, patients receiving tight glycaemic control had accelerated weaning from mechanical ventilation and accelerated discharge. Subsequently tight glycaemic control was shown significantly to accelerate resolution of infection ${ }^{21}$ and inflammation, ${ }^{23}$ to prevent nosocomial infection ${ }^{24}$ and to reduce catabolism. ${ }^{2526}$

Benefits of tight glycaemic control for critical illness have become less clear with the recent publication of NICE-SUGAR. ${ }^{27}$ This large RCT, performed in adults expected to require ICU treatment for $\geqslant 3$ days, found that intensive blood glucose control (4.5-6.0 mM) increased mortality (odds ratio 1.14, 95\% CI 1.02 to 1.28) compared with conventional glucose control ( $\leqslant 10 \mathrm{mM}$ ). A meta-analysis of 26 studies including these three large RCTs found no difference in mortality with tight or conventional glycaemic control. ${ }^{28}$ Tight glycaemic control was associated with a sixfold increased risk of hypoglycaemia which may have offset beneficial effects of glycaemic control and insulin therapy. The optimal strategy for management of hyperglycaemia in ICU patients is now unclear, and more moderate glycaemic control (blood glucose 8$10 \mathrm{mM}$ ) is currently recommended. ${ }^{29}$

Despite recent ICU findings, there remains a potential case for testing glycaemic control in patients with COPD with exacerbations requiring hospitalisation. Oral corticosteroids are a key component of exacerbation management in COPD, and subgroup analysis of NICESUGAR suggests heterogeneity of response to glycaemic control in those receiving steroids. ${ }^{29}$ Infection, inflammation and muscle weakness are prominent components of COPD exacerbations and all are improved by glycaemic control with insulin. Most patients with COPD exacerbations, even those requiring NIV, are less critically unwell than those in ICU studies and may be more resistant to the detrimental effects of hypoglycaemia. 
Tight glycaemic control can be implemented in the acute medical unit outside the intensive care setting with similar efficacy and risks of hypoglycaemia as those seen in ICU studies. ${ }^{30}$ However, optimal glycaemic control in COPD remains unclear and ICU studies have shown us that intensive strategies should not be adopted without rigorous testing in multicentre clinical trials.

In their paper, Chakrabarti and colleagues wisely steer clear of discussing the sticky issue of glycaemic control during COPD exacerbations. Rather they concentrate on the interesting finding that blood glucose $<7 \mathrm{mM}$ in combination with respiratory rate $<30 / \mathrm{min}$ predicted a successful outcome from NIV in $97 \%$ of patients with acute hypercapnic respiratory failure. Treatment failure was more likely (but not inevitable) in those with respiratory rate $>30 / \mathrm{min}$ and hyperglycaemia. If their findings are confirmed in other patient cohorts, this simple prognostic index could support discussions with COPD patients and their families prior to initiation of NIV and allow targeting of more intensive monitoring and treatment for those at greater risk of NIV failure. However, measurement of blood glucose is not currently part of NICE/BTS (National Institute for Health and Clinical Excellence/British Thoracic Society) guidelines for the management of COPD exacerbations, ${ }^{31}$ and data about blood glucose were not collected in the 2008 national COPD audit. This paper adds to the growing evidence that hyperglycaemia is important during COPD exacerbations and supports inclusion of blood glucose assessment in future recommendations. However, it does not clarify underlying mechanisms or make the case for tight glycaemic control, both of which require further study.

\section{Competing interests: None.}

Provenance and peer review: Commissioned; not externally peer reviewed.

\section{REFERENCES}

1. Baker EH, Wood DM, Brennan AL, et al. Hyperglycaemia and pulmonary infection. Proc Nutr Soc 2006;65:227-35.

2. Capes SE, Hunt D, Malmberg K, et al. Stress hyperglycaemia and increased risk of death after myocardial infarction in patients with and without diabetes: a systematic overview. Lancet 2000;355:773-8.

3. Capes SE, Hunt D, Malmberg K, et al. Stress hyperglycemia and prognosis of stroke in nondiabetic and diabetic patients: a systematic overview. Stroke 2001;32:2426-32.

4. Yendamuri S, Fulda GJ, Tinkoff GH. Admission hyperglycemia as a prognostic indicator in trauma. J Trauma 2003;55:33-8.

5. McAlister FA, Majumdar SR, Blitz S, et al. The relation between hyperglycemia and outcomes in 2,471 patients admitted to the hospital with community-acquired pneumonia. Diabetes Care 2005:28:810-5.

6. Baker EH, Janaway $\mathrm{CH}$, Philips BJ, et al. Hyperglycaemia is associated with poor outcomes in patients admitted to hospital with acute exacerbations of chronic obstructive pulmonary disease. Thorax 2006:61:284-9.

7. Ram FS, Picot J, Lightowler J, et al. Non-invasive positive pressure ventilation for treatment of respiratory failure due to exacerbations of chronic obstructive pulmonary disease. Cochrane Database Syst Rev 2004;(3):CD004104.

8. Moretti M, Cilione C, Tampieri A, et al. Incidence and causes of non-invasive mechanical ventilation failure after initial success. Thorax 2000;55:819-25.

9. Chakrabarti B, Angus RM, Agarwal S, et al. Hyperglycaemia as a predictor of outcome during non invasive ventilation in decompensated COPD. Thorax 2009;64:857-62

10. Adrogue HJ, Chap Z, Okuda Y, et al. Acidosis-induced glucose intolerance is not prevented by adrenergic blockade. Am J Physiol 1988;255:E812-23.

11. Esposito K, Nappo F, Marfella R, et al. Inflammatory cytokine concentrations are acutely increased by hyperglycemia in humans: role of oxidative stress. Circulation 2002;106:2067-72.

12. Andreozzi F, Succurro E, Mancuso MR, et al. Metabolic and cardiovascular risk factors in subjects with impaired fasting glucose: the 100 versus $110 \mathrm{mg} / \mathrm{dL}$ threshold. Diabetes Metab Res Rev 2007;23:547-50.

13. Choi KM, Lee J, Lee KW, et al. Comparison of serum concentrations of C-reactive protein, TNF-alpha, and interleukin 6 between elderly Korean women with normal and impaired glucose tolerance. Diabetes Res Clin Pract 2004;64:99-106.

14. Loukides S, Polyzogopoulos D. The effect of diabetes mellitus on the outcome of patients with chronic obstructive pulmonary disease exacerbated due to respiratory infections. Respiration 1996;63:170-3.

15. Philips BJ, Redman J, Brennan A, et al. Glucose in bronchial aspirates increases the risk of respiratory MRSA in intubated patients. Thorax 2005;60:761-4.

16. Brennan AL, Gyi KM, Wood DM, et al. Airway glucose concentrations and effect on growth of respiratory pathogens in cystic fibrosis. J Cyst Fibros 2007;6:101-9.

17. Brennan AL, Baines DL, Woollhead A, et al. Development of an in vitro model to investigate the effect of glucose on the interaction between respiratory epithelia and bacterial pathogens. Thorax 2006;61(Suppl II):ii101

18. Spruit MA, Gosselink R, Troosters T, et al. Muscle force during an acute exacerbation in hospitalised patients with COPD and its relationship with CXCL8 and IGF-I. Thorax 2003:58:752-6.

19. Martinez-Llorens JM, Orozco-Levi M, Masdeu MJ et al. [Global muscle dysfunction and exacerbation of COPD: a cohort study]. Med Clin (Barc) 2004;122:521-7.

20. Vanhorebeek I, De VR, Mesotten D, et al. Protection of hepatocyte mitochondrial ultrastructure and function by strict blood glucose control with insulin in critically ill patients. Lancet 2005:365:53-9.

21. Van den Berghe G, Wouters P, Weekers F, et al. Intensive insulin therapy in the critically ill patients. N Engl J Med 2001;345:1359-67.

22. Van den Berghe G, Wilmer A, Hermans G, et al Intensive insulin therapy in the medical ICU. N Engl J Med 2006;354:449-61.

23. Hansen TK, Thiel S, Wouters PJ, et al. Intensive insulin therapy exerts antiinflammatory effects in critically ill patients and counteracts the adverse effect of low mannose-binding lectin levels. J Clin Endocrinol Metab 2003;88:1082-8.

24. Hemmila MR, Taddonio MA, Arbabi S, et al. Intensive insulin therapy is associated with reduced infectious complications in burn patients. Surgery 2008;144:629-37.

25. Ellger B, Richir MC, Van Leeuwen PA, et al. Glycemic control modulates arginine and asymmetricaldimethylarginine levels during critical illness by preserving dimethylarginine-dimethylaminohydrolase activity. Endocrinology 2008:149:3148-57.

26. Biolo G, De CM, Lorenzon S, et al. Treating hyperglycemia improves skeletal muscle protein metabolism in cancer patients after major surgery. Crit Care Med 2008;36:1768-75.

27. Finfer S, Chittock DR, Su SY, et al. Intensive versus conventional glucose control in critically ill patients. $N$ Engl J Med 2009;360:1283-97.

28. Griesdale DE, de Souza RJ, van Dam RM, et al. Intensive insulin therapy and mortality among critically ill patients: a meta-analysis including NICE-SUGAR study data. CMAJ 2009:180:821-7.

29. Bellomo R, Egi M. What is a NICE-SUGAR for patients in the intensive care unit? Mayo Clin Proc 2009;84:400-2.

30. Archer JA, Misra S, Getov S, et al. Feasibility and safety of tight glycaemic control outside the intensive care setting. Br J Clin Pharmacol 2009; in press (abstract).

31. National Collaborating Centre for Chronic Conditions. Chronic obstructive pulmonary disease. National clinical guideline on management of chronic obstructive pulmonary disease in adults in primary and secondary care. Thorax 2004;59(Suppl 1):1-232.

\section{Identification of those at risk after acute pulmonary embolism}

\section{Andrew J Fisher, Paul A Corris}

Northern Pulmonary Vascular Unit, Freeman Hospital and Institute of Cellular Medicine, The Medical School, Newcastle University, Newcastle upon Tyne, UK
Correspondence to: Professor A J Fisher, Institute of Cellular Medicine, The Medical School, Newcastle University, Newcastle upon Tyne NE2 4HH, UK a.j.fisher@newcastle.ac.uk
It is well recognised by hands-on clinicians that patients who present acutely with haemodynamic compromise and hypotension with a systolic pressure of $<90 \mathrm{~mm} \mathrm{Hg}$ due to acute pulmonary embolism (PE) have a poor prognosis. This is reflected in current British Thoracic Society guidelines in the management of acute PE which recommend 
aggressive interventions in this population, with thrombolysis as first-line treatment. ${ }^{1}$

However, stratification of mortality and morbidity risk in normotensive patients who present with acute $\mathrm{PE}$ is less clear. Demonstration of right ventricular dysfunction or dilatation on either echocardiography or spiral HRCT scanning of the chest has been used as a potential indicator of those requiring more aggressive treatment even if normotensive. Immediate availability of these modalities is not universal, and a simpler way of identifying those patients at highest risk of adverse events and mortality remains critical as the inpatient mortality rate for normotensive patients with acute $\mathrm{PE}$ is approximately $10 \%{ }^{2}$ A number of previous studies have attempted to identify biomarkers which have prognostic power in patients with acute PE. Many of these studies have focused on cardiac biomarkers including the troponins and natriuretic peptides which correlate with right ventricular dysfunction on echocardiography. ${ }^{3}$ Whether these systemic biomarkers correlate with an increased risk of poor outcome in patients has been the subject of debate and, at present, there is no clear consensus as to the predictive value of cardiac biomarkers as a guide to risk stratification in this population.

The recent European Society of Cardiology guidelines on the diagnosis and management of acute pulmonary embolus published in 2008 have gone as far as including raised levels of the B-type natriuretic peptides BNP or NT-proBNP and increased cardiac troponins as markers of increased risk of a poor outcome due to PE-related mortality. ${ }^{4}$

In this issue of Thorax, Lega and colleagues $^{5}$ from Quebec, Canada and Lyons, France present a very comprehensive and methodologically robust metaanalysis of the prognostic value of natriuretic peptides and troponins in acute PE (see page 869). This is an extremely important piece of work, which brings together results of a large number of studies in this area. In addition to identifying all published studies capable of evaluation, the authors went to great lengths to minimise publication bias by examining all the abstract presentations at major international respiratory meetings over the last 10 years. In this metaanalysis, the methodological quality of the included studies was systematically reviewed to look for any evidence of selection bias, information bias, where the results of cardiac biomarker measurement influenced management and confounding bias and where other diseases such as renal failure or chronic heart failure may have influenced a result. One of the difficulties in comparing the results of the included studies is the range of different assays used to measure the natriuretic peptides. Four different assays were identified and different studies used different thresholds to define positive and negative results. In many, the definition of a positive or negative result was made at the end of the study based on receiver operating curve analysis. Despite these limitations, the results show that raised levels of B-type natriuretic peptides are associated with an increased risk of mortality, serious adverse events and right ventricular dysfunction after PE. This association also importantly appears to hold true for both haemodynamically stable and unstable patients at presentation. It also appears to be robust even in the presence of co-factors such as chronic heart failure and renal failure. The metaanalysis did, however, suggest that BNP measurement appeared to be more accurate than NT-proBNP in correlating with right ventricular dysfunction.

A previous meta-analysis has been published in this area. Work by Becattini and colleagues showed that positive troponins correlated with right ventricular myocardial damage and with poor outcomes after acute PE. ${ }^{6}$ The paper by Lega and colleagues adds to the existing literature by the robustness of its analysis and the careful search for studies. Specifically, this meta-analysis also considered the combined benefit of troponin and natriuretic peptide measurement. When a combination of natriuretic peptide and troponin measurement is used to stratify risk, the study shows that, in patients with an increase in natriuretic peptide, additional elevation of troponins is associated with a further increase in PE-related mortality and serious adverse events. Importantly, this held true when haemodynamically stable patients were considered for all-cause mortality with an odds ratio of 6.9 (confidence interval 2.3 to 20.7) and acute PE-related mortality odds ratio 8.4 (confidence interval 2.1 to 33.4 ).

This study offers very useful insights into risk stratification in normotensive patients presenting with acute PE. Its timing is particularly important as there is growing interest in the outpatient management of individuals presenting with acute PE who are haemodynamically stable. In light of the still high hospital mortality in this group, it is important that every effort is made to identify those at the highest risk who would be unsuitable for outpatient management. The measurement of natriuretic peptides and troponins in this population may not only be able to identify those patients at highest risk but also, conversely, if the natriuretic peptide level is normal, provide reassurance that a lower risk patient is eligible for outpatient management. This is very much the case with supportive evidence from this study showing that "natriuretic peptides are particularly helpful in identifying low-risk patients".

There are caveats on the conclusions drawn from this meta-analysis. It is important to recognise that the conclusions give the rationale for a large-scale prospective multicentre randomised trial of cardiac biomarkers to stratify risk in PE. Possible interventions in such a study could include early thrombolysis in haemodynamically stable patients that have yet to undergo formal assessment for right ventricular dysfunction, inpatient anticoagulation and close observation and outpatient anticoagulation.

The authors should be congratulated on their valuable contribution to this important debate, and it is hoped those involved in research into acute PE will take this information forward in the design of clinical trials to provide some more definitive answers linking risk stratification, intervention and prospective outcomes in this group of patients.

Competing interests: None.

Provenance and peer review: Commissioned; not externally peer reviewed.

Thorax 2009;64:832-833. doi:10.1136/thx.2009.114009

\section{REFERENCES}

1. British Thoracic Society. BTS guidelines for the management of suspected acute pulmonary embolism. Thorax 2003:58:470-84.

2. Bova C, Pesavento R, Marchiori A, et al. Risk stratification and outcomes in hemodynamically stable patients with acute pulmonary embolus: a prospective, multi-centre, cohort with three months follow-up. J Thromb Haemost 2209; 7:938-44

3. Kostrubiec M, Pruszczyk $P$, Bochowicz $A$, et al. Biomarker-based risk assessment in acute pulmonary embolism. Eur Heart J 2005;26:2166-72.

4. Torbicki A, Perrier A, Konstantinidis S, et al. Guidelines on the diagnosis and management of acute pulmonary embolus. European Society of Cardiology. Eur Heart J 2008;29:2276-315.

5. Lega J-C, Lacasse Y, Lakhal L, et al. Natriuretic peptides and troponins in pulmonary embolism: a meta-analysis. Thorax 2009;64:869-75.

6. Becattini C, Vedovati MC, Agnelli G. Prognostic value of troponins in acute pulmonary embolus: a metaanalysis. Circulation 2007;116:427-33. 